\title{
Reversing autism: Longitudinal data from an ongoing autism intervention study
}

\author{
Steven Evans ${ }^{1 *}$ and Debra Jude Fuller ${ }^{1,2}$ \\ ${ }^{1}$ Therapeutics Research Institute, Omaha, Nebraska 68131, USA \\ ${ }^{2}$ Myrrh Foundation, Baltimore, MD 21201, USA
}

\begin{abstract}
Building on previously reported results, this study presents longitudinal data involving the implementation of a single, safe, over-the-counter food supplement previously identified and shown in a rigorous clinical trial to significantly improve outcomes among participants with autism spectrum disorder. Six month outcomes for the supplement use demonstrated a $42 \%$ reduction in a battery of attributes expressed at the severe or moderately serious level (to no problem or a slight problem level). Current (but sparse) data demonstrate a 59\% reversal at the 12 -month period. A second parallel combination strategy that combined the supplement with the use of a vibrational medicine device yielded even more remarkable improvements with a $74 \%$ reversal in six months and an $89 \%$ reversal after 12 months. Without any side effects, and with either a very inexpensive and safe one-a-day capsule supplement or a quite low-cost device in combination, either strategy continues to demonstrate a powerful and positive intervention for individuals with autism spectrum disorder. These include males and females, ages three to 33 , some with significant autism behaviors for more than 25 years. The Therapeutics Research Institute continues to add participants and will continue to accrue data for the foreseeable future as long as the (positive) outcomes warrant.
\end{abstract}

\section{Background}

This report will summarize longitudinal data regarding an ongoing intervention study for individuals with autism spectrum behavior. It is a follow-up to a prior report [1] which described the beginning of this Study using a combination strategy which we will shortly but briefly review below. That Study by Evans and Fuller in turn was initiated and prompted by yet a prior clinical trial [2] providing a simple, safe, over-the-counter food supplement, which yielded significant positive outcomes after a 18-week period.

As previously reported by Evans and Fuller, their combination intervention yielded an approximately $39 \%$ reduction in presenting attributes of the autism cohort which compared favorably to the approximately $34 \%$ reduction obtained by the trial noted. It may be noted that these two figures are not really comparable since different assessment methods were employed in each case.

\section{Purposes}

The purpose of this extended study is to address a number of key, new issues raised by the prior reported intervention study. First, would the results of the prior study be maintained over a longer period of time? By analogy, we know that the benefits of the levodopa drug intervention for Parkinson's begin to diminish over time so might this be true of this intervention also? A second important and closely related question is whether the six-month period reported by Evans and Fuller represented a peak point of benefit or might benefits continue to accrue over time? That is, just how long might improvement continue as the intervention continues? In concert, the approximate level of benefit by both prior reports of $34 \%$ and $39 \%$ may be viewed as roughly in the same ballpark even if there were not similar measures of outcome results so we may ask whether there is also a yet higher level of reversal of attributes if the intervention continues. This report will address all of these added issues.
There is a second important contribution this current report provides. One of the assessment instruments used initially in the noted trial lists 58 attributes a subject may exhibit and provides four levels at which that attribute may express itself from "no problem" to a "slight problem" to a "moderately serious" level to a final "severe" level. So, there is a total of 232 possible data points any one subject might exhibit in any one reporting period. Over a 12-month period, in theory any one of 2,784 values might arise. One of our challenges was to impose a reasonable reduction in this data universe so that we might be able to provide a useful, insightful and reasonably reflective measure for each client as they progressed through each month of assessment and enable us to both reflect the client's progress as well as compare the progress among all the clients.

We believe we have accomplished this and will describe our approach in the sections below. We note that with our addition of more and more clients each month to the Study under differing arms of interventions (a single supplement intervention and a combination intervention arm), this reporting methodology has been most instrumental to track progress and will be used henceforth as we continue to accrue and then report yet more data over significantly longer and longer periods of time. As we will exemplify with the data collected so far, we do not as yet believe the upside potential of this intervention study has been reached so that this ongoing analysis will continue to be warranted.

Lastly we observe that the final purpose of this ongoing study is to aid individuals with autism. This is one reason we elected to refer to it as an intervention study rather than a trial - we wish to

Correspondence to: Steven Evans, Therapeutics Research Institute, Omaha, Nebraska 68131, USA, E-mail: sevans@gsm-usa.com

Received: May 23, 2017; Accepted: June 05, 2017; Published: June 09, 2017 
help these individuals and their families better address their autism behaviors. For this reason, we will not create a placebo arm nor plan to end the study in any specific future time. With the goal to provide a worthwhile service, neither of these two actions would be justified. It is our best assessment from the past and ongoing data that the time for further "trials" has ended, even though we recognized some will initiate an unending call for more (trial) data, for more research. Such is the more common practice in medical research. We do not ascribe to this view. The current data is relatively strong and positive as we shall present, and the downside is minuscule with this very inexpensive and extremely safe food supplement. To reiterate the observation first made in the clinical trial, this food supplement has an extremely low toxic profile. Hence this study is part of an ongoing intervention to an ever-larger audience of participants.

Finally we would offer the view that given all the data in the aggregate currently available, the prospect to have an autism family keep their child on a placebo for one or more years is ill-conceived and unsustainable. When this view is combined with the outcome data we shall present, we believe it is the most ethically appropriate choice to provide this intervention and simply monitor outcomes. For this reason, we also did not invoke formalistic ties with other medical institutions since no medical trial as such is underway. TRI researchers have decades of experience in designing and implementing clinical trials and supplement studies when previously associated with medical schools and draw on this expertise if and when needed. Here we are engaged with what has been called grassroots science [3] where a formal translation is made from research to practice and then acted upon by participating individuals albeit TRI provides expert oversight over all participants as they provide monthly feedback.

\section{Methods and design}

\section{Supplement}

The Therapeutics Research Institute (TRI) buys the supplement off the Internet from a commercial manufacturer. Its suitability as a possibly viable candidate has been previously documented [1]. The original trial used a purified sulforaphane extract exclusively provided for their trial. However, from our own research, we deduced that this supplement could be essentially replicated with an extremely careful and suitable selection from similar, available over-the-counter products. We believe our data further confirms our judicious selection of such a product. This report as well as the prior study provide very useful and independent data that this selection represents a worthwhile intervention.

The original trial [2] modulated dosage based on weight - although there was no a priori basis for this choice other than it might appear to be useful to increase dosage based on the greater mass of the participant. We however utilize a single capsule a day which has proven to be quite appropriate. Since the extract of sulforaphane passes the blood-brain barrier and likely acts there to achieve its effect, we observe that brain size does not differ even when body weight may vary immensely. Hence the same dose of one daily capsule was used irrespective of body weight.

Another important feature about this commercial product is that future client usage is not dependent on any tie to TRI. To maintain a maximum ethical stance, we buy it for all clients and provide it without charge. So, we sell nothing nor have any charges or fees nor any financial tie-ins. Being an available over-the-counter commercial product, we also do not seek any intellectual property claims, so this further keeps the study's data collection insulated from product (and hence data) bias. The supplement represents for some participants the single intervention provided.

\section{The Bio-resonance (Rife) intervention}

In this more controversial component to our combination strategy, we added a second intervention. From our purpose described above to achieve the greatest possible positive impact on the autism population, we elected to add the use of a vibrational medicine or bio-resonance device. Some will not have recognized this strategy and so we will recapitulate some background on it to aid the uninitiated reader.

There has been recent growing awareness in the USA of the use of bio-resonance to address disease states. Although initially dismissed in the USA like many other non-traditional modalities such as acupuncture or even chiropractry in prior years, there has come begrudging acceptance here even though it has much more widespread use in Asia and Europe. To provide some concrete advances defining its use, vibrational medicine to treat brain cancer has been officially approved by the USA FDA after clinical trials demonstrated its effectiveness. For brain cancer, the first clinical trial obtained FDA approval for its use with recurrent brain cancer while a second FDAreviewed trial approved its use for primary brain cancer [4]. Front page news in Omaha, Nebraska [5] described how one somewhat famous business leader was using it for his treatment. It was revealing that even though this intervention was FDA-approved after clinical trials demonstrated statistically significantly positive outcomes, medical researchers expressed their hesitation to use such a "sci-fi" intervention [6].

This reaction no doubt can be expected to be mainstream even though one device manufacturer indicates a wi-fi version has been used by Cosmonauts on the space station since "office visits" there can be somewhat prohibitive while these small portable devices can be programmed to address more than 3500 disease states. Some of these devices are known as Rife machines named after their most famous American inventor.

We supplied such a Rife machine to some of the participants along with the supplement as a combination intervention while others have used just the supplement. The most longitudinal data is from those with the joint combination intervention which we present below while we are also accruing data on those with just the supplement intervention as well. Our initial intent was just to use the joint combination intervention since our purpose was to achieve the maximum impact on the autism cohorts.

However a lack of funding later required us to enroll those with use of the supplement alone. Hence in this report, we will provide available data so far on both these Study arms. We note in passing that the data currently supports our original design to use the joint combination since as we will see, the outcomes are uniformly better. Still, we shall see the supplement-alone group also exhibited strong positive gains.

In providing the bio-resonance device, we elected to purchase the Rife Professional online from healthproducts2.com in combination with their pad which conveys the vibrations powered by the Rife unit and both were provided by TRI (without charge) to a number of the participants. Using this programmable Rife device, we currently program in seven different programs designed to address seven different possibly etiological factors. These factors include a listing for autism itself, heavy metals detox, viral and fungal pathogens, systemic inflammation, and gram-positive bacteria. 
A web search on Rife machines will bring the reader to a number of sites showing how the right vibration can cause the destruction of a parasite with that exact same frequency signature while no other tissue or organs are affected otherwise. It is useful to note that a recent publication involved a clinical trial in which 11 children with autism were give three weeks of the antibiotic vancomycin, and the authors reported a subsequent $80 \%$ reduction in autism attributes. At the simplest level, vancomycin destroys gram-positive bacteria. Although to date many autism researchers are loathe to associate any bacterial agent with the etiology of autism, with our approach we need not confront this contentious issue. We simply program one of the seven Rife settings to address gram-positive bacteria, and if there is benefit, it accrues. If not, no harm arises.

\section{Enrollment population}

We have accrued participants from disparate sources and at quite disparate times. The ages run from 3 to 33 involving both males and females. The data reported from these participants are not always accrued uniformly every month since virtually all participants have exhibited reporting lapses. For this report, we will present all available data for five participants on the combination strategy and five participants on the supplement-only strategy. The current report covers the outcomes from those who exhibited as few as 5 attributes at a moderately serious or severe level among the 58 attributes itemized on the Aberrant Behavior Checklist [7] up to individuals exhibiting 40 of the attributes at a severe or moderately serious level.

\section{Results}

\section{Data tabulation}

The way we have found to encapsulate the vast amount of data collected is to focus on just those attributes expressed at a moderately serious or severe level. The attributes that occurred at a slight problem level that might later disappear at the no problem level did not present a particularly germane issue. Similarly, attributes that were at a no problem level that might move to a slight problem level (and back again) again did not give a useful summary of the participants' state of progress (or loss thereof). Hence, we have elected to take the number of attributes (among the 58 on the Aberrant Behavior List) that were presenting at a moderately serious or severe level as the baseline measure for a participant. Then we compared each subsequent report they provided to count the number of current attributes at a moderately serious or severe level they had eliminated ("reversed") as compared to the baseline total.

This ratio of the total number of reversed moderately serious or severe level attributes as compared to the baseline presentation of attributes at those two levels gives a most useful measure of any progress an individual is making and similarly allows an excellent thumbnail comparison among participants. As one more convention, given the lack of continuity in some participant's monthly data reporting we received, figures shown are the closest available outcomes following the cut-off date for each respective column.

\section{Longitudinal data}

Presented below are the data available so far for the two intervention strategies of (1) the combination intervention of both the use of a Rife plus the supplement and (2) the supplement alone. We have indicated where the participant failed to report data (d.n.a.-data not available) or where the participant has not as yet provided the data (n.y.a.-data not yet available). As explained above, the ratios shown describe the number of attributes reversed as defined above compared to the total number of moderately serious or severe attributes the participant first presented. The percent figures round off the ratios to a simple two figures with .5 rounded up. These selections allow the reader to quickly identify the trends that the data reflect (Table 1 ).

There are some key outcomes to emphasize in the data reported above. For the five participants who used both interventions for at least one year, the average outcome was an $89 \%$ reduction in severe or moderately serious attributes reported to either no problem or a slight problem. For clients taking just the supplement, after six months, the average severe or moderately serious attribute reduction was $42 \%$. This compares to approximately $34 \%$ reduction after about four months as reported in the initial Hopkins-Harvard-related trial, although we emphasize the comparison between their outcomes and ours is merely approximate since different comparison methodologies were actually used by each research group.

With the combination strategy, much more noticeable progress was usually evident in the first month while much less so for the supplement-only group. It was even harder to behaviorally perceive lower levels of improvement so the supplement-only group was advised to be much more patient than the combination strategy participants.

Over time, the combination group made much more progress much more quickly. Still, by six months, we see the supplement group approaching a reversal of about $42 \%$ of the significant attributes. For the combination group, they are reversing about $74 \%$ of the important attributes. By the 12th month, the combination group is reversing about $89 \%$ of the total of attributes with which they initially presented.

In the combination group, note particularly the linear and systematic progress being made. Although not totally perfect, this adds to the confidence that we are seeing a very uniform and systematic improvement in the individuals. Recognizing the data available is still sparse, nonetheless its uniformity adds to the initial confidence about the likely impact each strategy is having. Even for individual \#4 where there was a downturn in progress between the sixth and twelfth month, the participant was off product for about two months due to a lack of communication. Even this relapse is consistent with the experience described in the first trial where the discontinuance of the supplement led to a loss of progress. Irrespective of the temporary loss of continuity, we reported the data as received but suggest this reinforces the case for

Table 1. Reductions in Severe or Moderately Serious Attributes. d.n.a.-data not available; n.y.a. - data not yet available. Ratios represent the number of severe or moderately serious attributes reversed to no problem or just a slight problem as compared to the total number of initially reported as severe or moderately serious behavioral attributes.

\begin{tabular}{|c|c|c|c|c|}
\hline \multicolumn{5}{|c|}{ Reductions in Severe or Moderately SeriousAttributes } \\
\hline \multicolumn{5}{|c|}{ Using Supplement + Rife Intervention } \\
\hline & 1 Month & 2 Months & 6 Months & 12 Months \\
\hline \multicolumn{5}{|c|}{ Clients } \\
\hline 1 & $11 / 17(65 \%)$ & $13 / 17(76 \%)$ & $13 / 17(76 \%)$ & $17 / 17(100 \%)$ \\
\hline 2 & $10 / 15(67 \%)$ & $7 / 15(47 \%)$ & $14 / 15(93 \%)$ & $15 / 15(100 \%)$ \\
\hline 3 & $32 / 40(80 \%)$ & $21 / 40(53 \%)$ & $13 / 40(33 \%)$ & $28 / 40(70 \%)$ \\
\hline 4 & $18 / 19(95 \%)$ & $18 / 19(95 \%)$ & $18 / 19(95 \%)$ & $16 / 19(84 \%)$ \\
\hline 5 & d.n.a. & $6 / 18(33 \%)$ & $11 / 18(61 \%)$ & d.n.a. \\
\hline \multicolumn{5}{|c|}{ Using Supplement Intervention Only } \\
\hline 6 & $31 / 29(-7 \%)$ & $7 / 29(24 \%)$ & $13 / 29(45 \%)$ & $17 / 29(59 \%)$ \\
\hline 7 & $7 / 25(28 \%)$ & d.n.a. & $12 / 25(48 \%)$ & n.y.a. \\
\hline 8 & d.n.a. & d.n.a. & $8 / 24(33 \%)$ & d.n.a. \\
\hline 9 & $5 / 5(100 \%)$ & n.y.a. & n.y.a. & n.y.a. \\
\hline 10 & $9 / 35(26 \%)$ & $8 / 35(23 \%)$ & n.y.a. & n.y.a. \\
\hline
\end{tabular}


the impact of the intervention on the participant.

In neither group were there any reported side effects. The Rife application which one author has used extensively and continuously [8] has no outward physiologic manifestations and in this as well as all other studies the authors have undertaken, there have been no side effects reported. The supplement is in effect a concentrated extract derived from broccoli sprouts and we have not received any indication of any side effects at all. We have encountered anxieties among professionals to whom we have presented that perhaps there should be a multi-year, multi-center multi-thousand cohort doubleblind, placebo-controlled randomized study to validate the safety of broccoli sprouts. This Study's authors would admonish those opinion leaders who place extreme concern with liability over a reasonable perspective. Sometimes things simply are not too complicated and do not require the quite excessive measures that might otherwise apply in more traditional pharmacologic settings especially involving synthetic substances; we are quite aware that not everything "natural" is safe, but we are dealing here with a food supplement derived from a food source which simply has an unbelievable low toxic profile, to repeat the first trial's authors. Concomitantly we argue the payoff is quite extreme so that the risk-reward ratio is utterly minuscule.

\section{Discussion}

An interesting feature as we extend our data collection is that some participants did respond to the supplement alone in a most pronounced way. For client \#9 above, in less than two months there was an enormous positive response. Although we are strictly focused on the hard data as defined above, we have received what can also be called "emotional" data (or testimonials) unsolicited but provided nonetheless from some of the moms who are supervising their children in this study. We will provide one such unsolicited response below since it adds a human dimension that the data alone cannot convey. This mom, after having her somewhat high functioning child (\#9) on the supplement for two months, wrote us the following:

\section{Steve!}

I sent back the paperwork regarding (her son) that you sent to me. I also wanted to provide some specific examples for you. I do see noticeable improvement!

1. When I interrupt (her son) when he is engrossed in his video games he is not as annoyed. In the past, he would yell at me and say stuff like" "you always want me to stop and ruin my game!" and it could go on for a bit. Now, he's still annoyed but if, say, I offer him food he'll usually turn me down with an abrupt "no" but then he stops himself and says "but thank you for asking me, mom." I was blown away the first time it happened and now it's his typical response to me.

2. Whenever we've been at a park or pool and played catch with (her son) in the past he would become very angry when other kids tried to play, too. He wanted the ball to always come to him and he didn't want either of us (or his older sister) to pay attention to the other kids. We were at a hotel pool about a month ago and my husband was throwing the ball to (her son) and after a little time, about 5 other boys were on (her son's) side of the pool trying to catch the ball. They were all jumping and diving for it at the same time. This went on for about two hours. Not only was (her son) sharing his dad and his ball but he would say "good catch" or "epic throw" to the other kids. He still said things like "that was a really bad throw" as well to the other kids but he also said it about himself if it wasn't good. I kept waiting for him to get angry and he didn't.

3. He's much more affectionate these days. I can even sneak a hug or kiss in public without him getting mad. Embarrassed, yes but not mad. He also really never wanted anyone outside of those closest to him to touch him. A few weeks ago he met some cousins of ours from Chicago that are older (70's) and wonderful people who paid a lot of attention to him. (Her son) really likes geography and these are well travelled people. We pulled out a globe and (her son) asked about a million questions. At the end of the night, without prompting, he gave both our cousins huge hugs and asked when he would see them again. It was awesome!

Thank you for allowing us to participate in your program! I am seeing notable differences and it's wonderful. If you would like additional information, please let me know.

\section{Warm}

regards,

(His Mom)

We have received a number of such commentaries that reinforce the story told by the data itself. In another instance, one of the teachers of a participant came by to tell of the remarkable transformation of the child in the study who she supervises during the day with special education services since the child could not function in the standard public school classroom. She offered to tape her commentary which we did and have as part of the data archives. She related how the child has been totally transformed in her special classes - from extremely problematic and requiring enormous and incessant supervision and guidance concerning his quite disruptive behavior throughout the day to one of great compliance, patience, and excellent social interplay with both the environment there as well as with the other "special ed" students. Indeed, this child no longer needs this environment, has been "mainstreamed" (i.e., put into the regular public school system) and is doing well there without any added special education resources.

Besides being another phenomenally successful outcome, this case portends very significant social and economic consequences. As the autism population continues to escalate, the demand for special services will arise accordingly, putting an ever-increasing burden on limited economic resources. Just as in the case above, as these participants no longer exhibit noteworthy social dysfunction and indeed can be accommodated by the traditional social institutions (e.g., the regular public school system classroom), the implied financial burden to the social system could be projected to decrease exponentially rather than rise geometrically. This would argue for support of a massive expansion of this study supported from the public domain. In the currently unlikely event this should occur, we will not fail to report this development in our next study update.

There is of course not just the public cost to provide proper support for those with pronounced autistic behaviors. The impact on each family structure and family dynamics is most profound. The email above hints at the improvement arising at this level, not caught in the data tabulation but relayed nonetheless from the parents of the participants.

We will underscore two more outcomes in the data presented. Client \#4 is a 33-year male who was diagnosed with autism 25 years ago. Beginning quickly and continuing on, he has reversed $95 \%$ of all 
his moderately serious and/or severe attributes for most of the study time. This provides some evidence that the impact in this case, the combination strategy, on those even with long-standing autism might still resolve favorably.

\section{Summary and conclusions}

The continuation of this study is very strongly reinforcing our expectations that a significant reversal of autism is possible and is arising from both the combination strategy we have implemented as well as the single supplement strategy we have provided. The outcomes remain consistent and reasonably uniform. The trends remain in place and continue over time. Progress continues.

For the supplement group, they continue to improve and so we do not as yet know what if any upside limit there might be. The continuation of the study as is ongoing should shed light on this issue. For the combination strategy group, nearly all are nearing a reversal of essentially all their significant attributes at baseline, and we will continue to monitor their progress to see if they hold these gains.

\section{Future directions of research}

As noted, we will continue to add to the single strategy (supplement) group without limit. It will require external funding to enable us to add to the combination strategy although additions are occurring from those who have read our prior study results and who already have a Rife device available. We merely re-program their current Rife for them or guide them to do it themselves.

In our prior report, we noted even more radically under-served populations particularly in Asia. We have made progress in our outreach efforts but much more work (and funding) is needed to begin this initiative.

The most significant advance we can provide is to enroll ever larger numbers of participants, track all the data as meticulously as we can, and continue to report ever longer longitudinal outcomes to explicate progress to date. This unfortunately entails securing significant external funding, particularly to support the combination strategy with its one-time Rife provision, but even to support the supplement strategy as our target family size moves to 300, then 3000 , then 30,000 and more. Not totally surprising, the simplicity of the intervention seems to hinder the capture of the imagination of funding agencies. Certainly, without question, the combination strategy with its use of a ("sci-fi") Rife device, as we indicated one physician noted, further complicates funding prospects. However, TRI has irrevocably committed its resources and energies to the expansion of this effort as long as the data indicate such effort is warranted.

To keep both participants and other interested researchers apprised of continuing study progress, we have posted a dedicated web site that is available - ControlAutismNow.org - where we will post the current study's progress as well as ongoing developments.

\section{References}

1. Evans S, Fuller DJ (2016) Initial outcomes from an autism treatment demonstration. Clin Med Invest 1: 16-19.

2. Singh K, Connors SL, Macklin EA, Smith KD, Fahey JW, et al. (2014) Sulforaphane treatment of autism spectrum disorder (ASD). Proc Natl Acad Sci U S A 111: 1555015555.[Crossref]

3. Sal Restivo (2005) Grassroots Science. Science, Technology, \& Society: An Encyclopedia, Oxford University Press, Oxford pp:175-181.

4. Ansstas G, Tran DD (2016) Treatment with tumor-treating fields therapy and pulse dose bebacizumab in patients with bevacizumab-refractory recurrent glioblastoma: a case series. Case Rep Neurol 8: 1-9.

5. Julie Anderson (2017) Oriental Trading CEO excited about the results he's seeing with device to treat brain tumors. Omaha World-Herald.

6. CBS (2017) Sci-fi' cancer therapy fights brain tumors, study finds, AP.

7. Marshburn EC, Aman MG (1992) Factor validity and norms for the aberrant behavior checklist in a community sample of children with mental retardation. J Autism Dev Disord 22: 357-373.

8. Evans S (2016) Case studies in bio-resonance applications, Therapeutics Research Institute, Omaha, NE pp: 3-5.

Copyright: (C2017 Evans S. This is an open-access article distributed under the terms of the Creative Commons Attribution License, which permits unrestricted use, distribution, and reproduction in any medium, provided the original author and source are credited. 